\title{
Experimental evidence of van der Pol-Fitzhugh-Nagumo dynamics in semiconductor optical amplifiers
}

\author{
Stéphane Barland, ${ }^{1,2, *}$ Oreste Piro, ${ }^{1}$ Massimo Giudici, ${ }^{1,2}$ Jorge R. Tredicce, ${ }^{1,2}$ and Salvador Balle ${ }^{1}$ \\ ${ }^{1}$ Institut Mediterrani d'Estudis Avançats, IMEDEA (CSIC-UIB), C/ Miquel Marquès, 21, E-07190 Esporles, Spain \\ ${ }^{2}$ Institut Non Lineaire de Nice (UMR 6618, UNSA-CNRS), 1361 Route des Lucioles, F-06560 Valbonne, France
}

(Received 2 April 2003; published 19 September 2003)

\begin{abstract}
Thermo-optical pulsing in semiconductor amplifiers is experimentally shown to correspond to a very common excitable scenario (the van der Pol-Fitzhugh-Nagumo system). Self-sustained oscillations appear in the sequence predicted by this simple dynamical model as we change either the injection level or the bias current. Periodic modulation of these parameters leads to the characteristic phase-locking structure. Furthermore, coherence resonance is observed when external noise is added to the system.
\end{abstract}

DOI: 10.1103/PhysRevE.68.036209

PACS number(s): 05.45.- a, 42.65.Sf

Nonlinear optical systems are excellent workbenches for dynamical systems theory. Recently, for instance, considerable theoretical and experimental efforts have been devoted to find optical excitable behavior similar to that found in biological [1,2], chemical [3], or electronic [4,5] systems. Experimental studies of excitability in optical systems have been performed for lasers with optical feedback [6,7], lasers with an intracavity saturable absorber [8], lasers with optical injection [9], and two-section distributed-feedback lasers [10]. Excitability in these systems arises in the vicinity of a homoclinic bifurcation [8-10] or close to an Andronov bifurcation $[6,7]$.

However, we are not aware of any experimental observation of excitability in optical systems, which corresponds to one of the fundamental paradigms for excitable behavior in biological, chemical, or electronic systems, the van der PolFitzhugh-Nagumo (VPFN) model $[4,11]$. The existence of such a scenario for optical systems was theoretically suggested in Refs. [12,13] when analyzing passive nonlinear étalons. These devices were originally proposed as basic units for all-optical computing, information processing, and storage $[14,15]$, due to the possibility of bistable response to the injected optical power [16]. Though, it is often found that the system does not exhibit bistability but self-sustained optical pulsations [17]. These pulsations have been explained as a thermally induced instability $[12,13,17,18]$. Similar regenerative optical pulsations have also been observed in optical parametric amplifiers [19].

In this work we experimentally show that thermo-optical pulsation in broad area semiconductor optical amplifiers (SOAs) can be mapped by the VPFN model. Varying either the injected field or the bias current, the system displays the same sequence of dynamical behaviors predicted by this model: stability, excitable regimes, and thermo-optical selfpulsations. The correspondence with the VPFN scenario is further checked by studying the system's response to different types of modulation. We find excitability and oscillatory phase locking under periodic excitations as well as coherence

\footnotetext{
*Present address: Istituto Nazionale di Ottica Applicata, Largo E. Fermi, 6, I-50125 Firenze, Italy.
}

resonance under additive external noise, in agreement with the theoretical predictions. In addition, the "reversed hysteresis" cycle sometimes reported in these type of systems [20] can be simply explained as phase locking of the regenerative oscillations to parameter modulation.

The experimental setup is schematically shown in Fig. 1. The amplifier is a commercial (Thorlabs L9801E3P1, 100 $\times 1 \mu \mathrm{m}^{2}$ ) multiquantum well edge-emitting laser operated at currents above transparency and below threshold ( $I_{t h}$ $=208 \mathrm{~mA}$ ), its gain peak occurring around $980 \mathrm{~nm}$ and with a longitudinal mode spacing of $31 \mathrm{GHz}$. The SOA is mounted on a heat sink (Thorlabs TCLDM9/B) for thermal stabilization (better than $0.01 \mathrm{~K}$ ) achieved through a temperature controller (Thorlabs TEC2000), and the current source (Thorlabs LDC500) is stabilized to better than 0.1 $\mathrm{mA}$. A tunable, thermally stabilized, high-power semiconductor laser at $\lambda \sim 980 \mathrm{~nm}$, with a spectral width below 2 $\mathrm{GHz}$ (instrumental limit of our Fabry-Perot optical spectrum analyzer) and optically isolated from the SOA with a Faraday isolator (better than $40-\mathrm{dB}$ isolation) provides the injection beam, which is injected along the SOA axis and is homogeneous over the transverse cross section of the SOA. The optical power of the injection beam is controlled by an acousto-optic modulator, attaining a maximum of $8.5 \mathrm{~mW}$ before the collimator. The output from the SOA is focused onto an avalanche photodiode, and all dioptric elements in the setup are antireflection coated, with reflectivities below $1 \%$.

As the optical injection frequency is changed, light amplification is observed in an interval of $12 \mathrm{GHz}$ within each free-spectral range of the SOA. The gain shows a strong nonlinear dependence on the input power, with the output beam having a single-lobed profile when the frequency of the injected beam is on the low-frequency range $(\approx 3 \mathrm{GHz})$ of the above interval. Fixing the frequency of the injected beam within this nonlinear amplification range and increasing the injection power, we observe the dynamical sequence shown in Fig. 2. In the upper-left panel, corresponding to low optical injection, the detected power is stable around a low level. As the power of the injection beam is increased (uppercenter panel), the output power starts pulsing. It remains at a low level for most of the time, but square pulses appear 


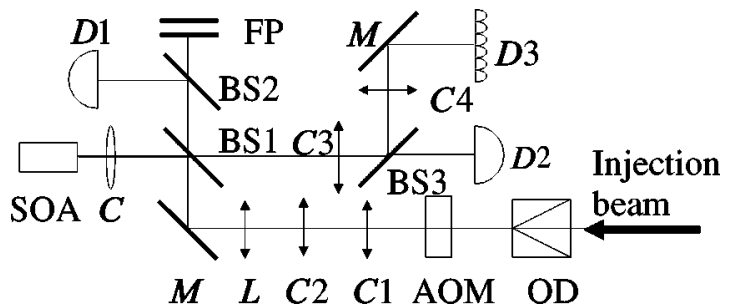

FIG. 1. Schematic of our experimental setup. OD: Optical diode, $C$ : collimator, Thorlabs C350TMB; AOM: acousto-optic modulator; $C 1-C 4$ : cylindrical lenses; $D 1$ and $D 2$ : avalanche photodiode; $D 3$ : photodetector array; $M$ : $99.9 \%$ mirrors; BS1: $10 \%$ transmission-90\% reflection, wedge beam splitter; BS2 and BS3: $50 \%-50 \%$ wedge beam splitters; FP: Fabry-Perot interferometer.

randomly. The pulse duration is almost uniform, while the interpulse times vary irregularly. Further increase of the injection (upper-right panel) makes the pulsing output more periodic. Still increasing the injected power leads to stable operation on a high power state through the reverse sequence, first spoiling the periodicity of the train of pulses and finally leading to stable behavior (see bottom panels). It is worth remarking that this dynamical sequence involves only the amplitude of the output beam, whose shape remains fixed. A similar dynamical sequence is obtained when the injected optical power is kept constant and the bias current is increased. The same dynamical sequences have also been observed in the detuning range 3-6 GHz. Although in this case the output profile displays three lobes, spatiotemporally resolved measurements showed that only the amplitude of the whole pattern evolves in time.

These self-pulsations are strongly reminiscent of those observed in passive optical systems [17-19], with a pulsation frequency $(\sim$ tens of $\mathrm{KHz})$ much slower than the intrinsic time scales of the optical and material variables. This time scale is characteristic for the thermal response of the device, and we infer that there must be temperature changes that break down the theoretically predicted bistability [21]. From the dynamical point of view, temperature is not anymore a parameter but it becomes a dynamical variable with its (slow) time scale.

The former sequence of dynamical behaviors is the same as found in the generic VPFN model with noise [4,11],

$$
\begin{gathered}
\frac{d x}{d t}=y+x-\frac{x^{3}}{3}+\sqrt{\eta} \xi(t), \\
\frac{d y}{d t}=-\varepsilon(a x+b+y),
\end{gathered}
$$

as the control parameter $b$ is varied. In the absence of noise $(\eta=0)$, the fixed points $\left(x_{s}, y_{s}\right)$ of the system are given by

$$
\begin{gathered}
y_{s}=\frac{x_{s}^{3}}{3}-x_{s}, \\
y_{s}=-\left(a x_{s}+b\right),
\end{gathered}
$$

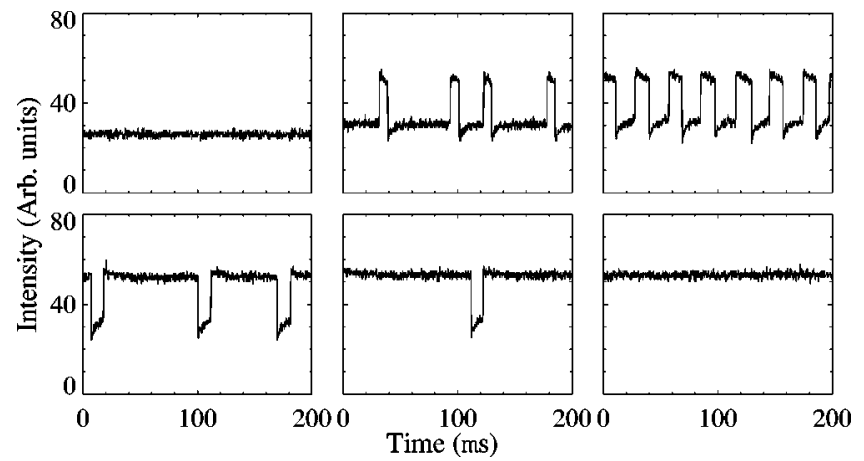

FIG. 2. Traces of the SOA output power for optical input power of (from left to right and top to bottom) $6.6 \mathrm{~mW}, 6.9 \mathrm{~mW}, 7.5 \mathrm{~mW}$, $8.2 \mathrm{~mW}, 8.5 \mathrm{~mW}$, and $8.7 \mathrm{~mW}$.

which have one real solution if $a>1-(3 b / 2)^{2 / 3}$, and three otherwise. For fixed $a>1$, the single fixed point is determined by $b$, and it is stable if $\left|x_{s}\right|>(1-\varepsilon)^{1 / 2}$ and unstable otherwise.

In the unstable regime the system exhibits oscillations which, for $\varepsilon \ll 1$, involve slow motion along the branches of positive slope of the $N$-shaped manifold $y=x^{3} / 3-x$, followed by fast jumps (where only $x$ changes) from one of the above branches to the other [22]. The oscillation frequency $f_{C}$ is determined by the control parameters $a$ and $b$ [23]. Then, the output of the system consists of a train of overshooting square-wave-like pulses as seen in the experiment. This pulse shape is characteristic of the VPFN system, and it is not compatible with the dynamical features of the other proposed scenarios for excitability.

In the stable regimes, a perturbation larger than a certain threshold triggers the emission of only one of the above pulses. The pulse shape is insensitive to the details of the stimulation, and the threshold is smaller the closer the system is to the instability point $\left|x_{s}\right|=(1-\varepsilon)^{1 / 2}$. Moreover, after escaping from the stable state the system returns to its vicinity through a deterministic trajectory in phase space; during this excursion, the system is insensitive to reasonably small perturbations, hence said to be in its "refractory time." These characteristics define the so-called "excitable" behavior. These "excitable pulses" may appear spontaneously when noise is added to the system, (i.e., $\eta \neq 0$ ), and then the escape rate from the stable state follows Kramers' law [24].

In Fig. 3 we plot typical time traces from Eqs. (1) and (2) as $b$ is increased. In Figs. 3(a) and 3(f), parameters are such that the system is in the stable regime and far away from the bifurcation points, so the time traces display only small fluctuations around the stable state. In Figs. 3(b), 3(c), and 3(e), the system is also set in the stable regime, but closer to the bifurcation points; in this case, the time traces show noiseinduced emission of excitable pulses, with rates that increase as the bifurcation point is approached [compare Figs. 3(b) and 3(c)]. Finally, in Fig. 3(d) parameters are set in the unstable regime, and the time trace consists in the aforementioned periodic train of squarelike pulses.

The sequence of behaviors as control parameters are changed, shown in Fig. 2, parallels that in Fig. 3. Both systems display a transition from a "low" to a "high" stationary 

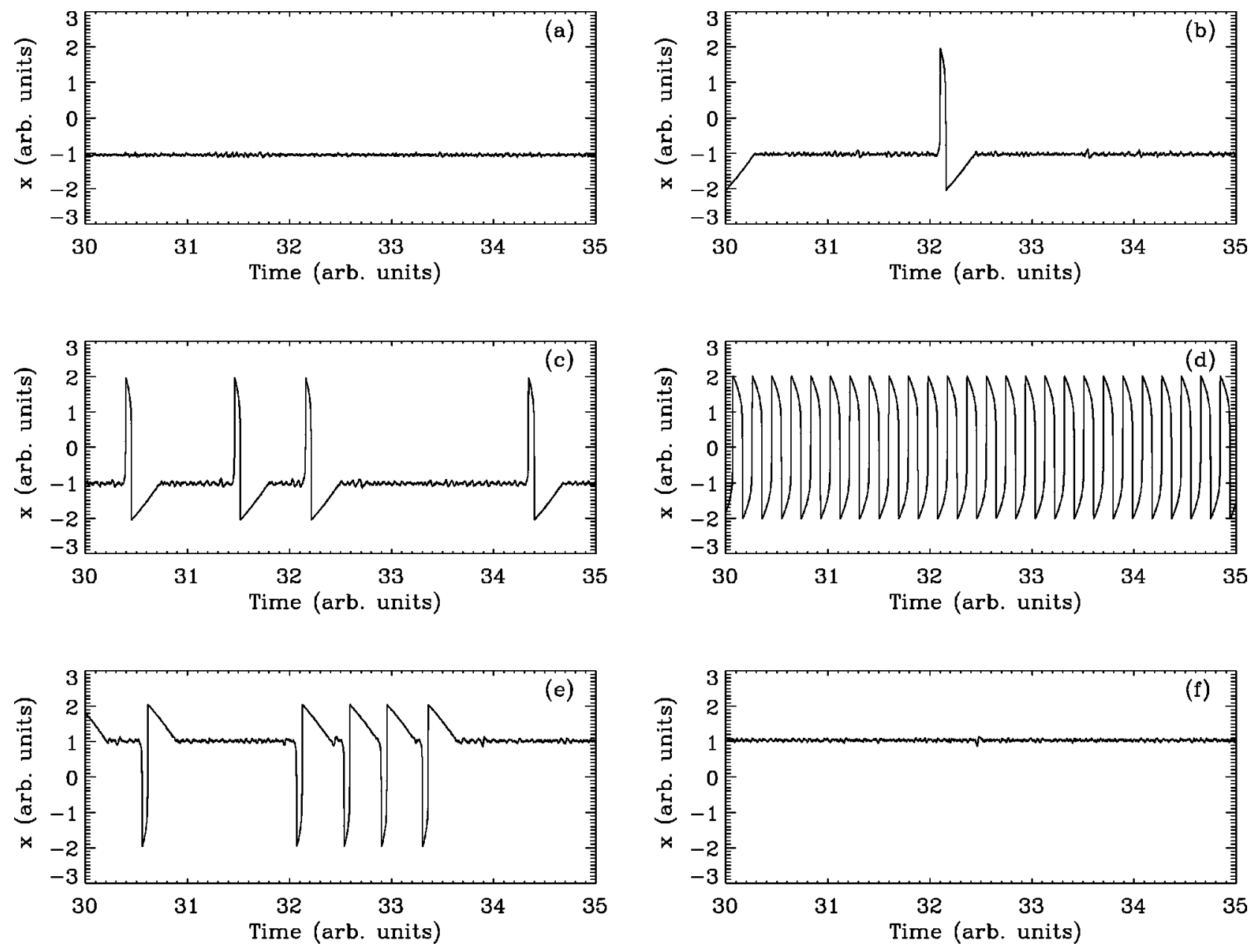

FIG. 3. Traces of the fast variable, $x$, in the VPFN model, for $\varepsilon=10^{-6}, a=10^{4}, \eta=10^{-4}$, and $b / a=1.05$ (a), 1.03 (b), 1.02 (c), 0.00 (d), -1.02 (e), and -1.04 (f).

state through an intermediate oscillatory regime. In both cases, the oscillations display two separate time scales, with abrupt jumps between stages of slow dynamics. Finally, in both cases we have regimes of noise-induced aperiodic pulsing, which occur close to the oscillatory regime, with a rate that increases as the bifurcation point is approached.

These similarities suggest that the dynamical scenario underlying the experimental system is that given by the VPFN model. This hypothesis can be tested by checking whether the experimental system possesses the dynamical properties of the model, namely, excitability and phase locking to external modulations.

The excitable character of the system can be tested by studying its response to external perturbations. We prepare the system in its low stable state and apply pulses of fixed duration and variable but small amplitude to the bias current. It is observed (see Fig. 4) that, although the maximum pulse amplitude is about $0.5 \%$ of the bias current, for pulse amplitudes below a critical value the response of the system is linear with the stimuli; however, above this critical value the SOA responds with a pulse of fixed amplitude and width. As a further check, we prepare the system in its low stable state and modulate the bias current with rectangular pulses of vari-

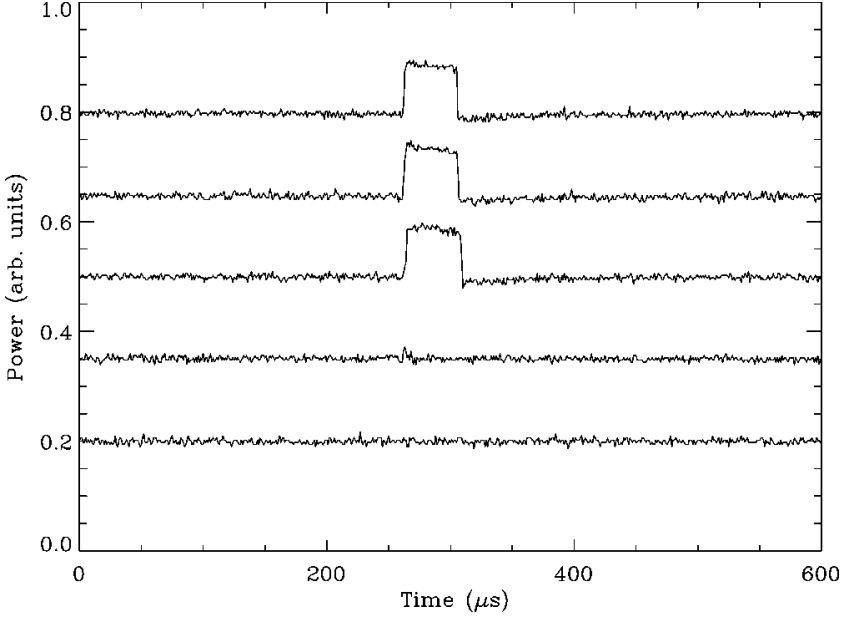

FIG. 4. Output pulses emitted in response to square pulses of $120 \mathrm{~ns}$ duration and different amplitudes applied to the bias current. From bottom to top: $0.47 \mathrm{~mA}, 0.66 \mathrm{~mA}, 0.73 \mathrm{~mA}, 0.80 \mathrm{~mA}$, and $0.92 \mathrm{~mA}$. The different curves have been vertically offset for clarity. The maximum amplitude of the applied pulses is $\sim 0.5 \%$ of the bias current. 


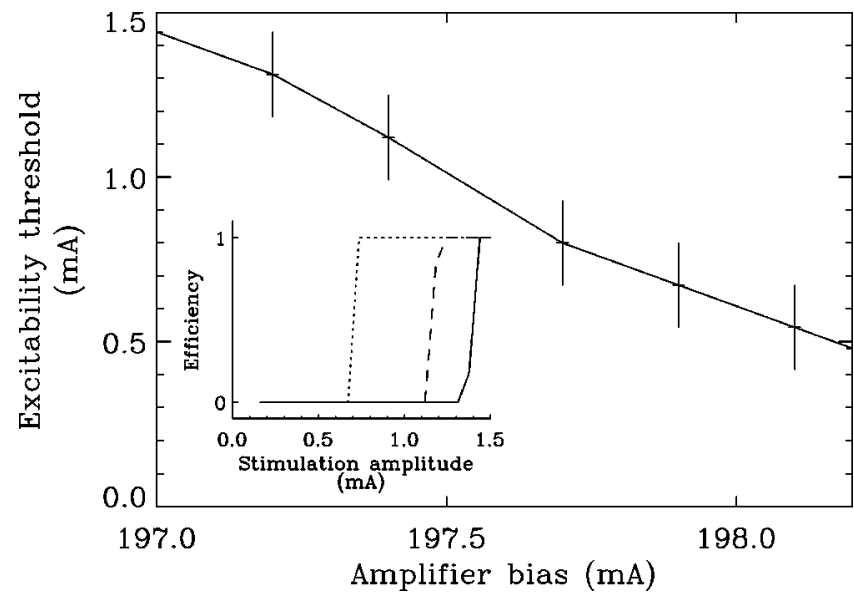

FIG. 5. Dependence of the threshold kicking amplitude on the amplifier's bias current. Inset: Pulse emission efficiency when the amplifier bias current is periodically kicked as a function of the kicking amplitude. Bias currents are $197.9 \mathrm{~mA}$ (dots), $197.4 \mathrm{~mA}$ (dashes), and 197.25 mA (solid).

able amplitude (below $1 \%$ of the bias current), 120 ns duration, and low repetition rate $(10 \mathrm{kHz})$. We measure the pulseemission efficiency, i.e., the ratio between the numbers of emitted and applied pulses, as a function of the amplitude of the stimuli. For amplitudes below a certain threshold the efficiency is nearly zero, becoming almost one for amplitudes above it (see inset in Fig. 5). As in the VPFN model, the threshold decreases almost linearly as the bias current approaches the point where the oscillatory regime sets in. Similar behavior is obtained when the external perturbation is applied to the acousto-optic modulator that controls the level of optical injection. These observations confirm the excitable character of our system. We stress that this behavior is found where the VPFN picture suggested that it should be, and that the dependence of the excitability threshold on the parameters is the same for the experimental system and the VPFN model.

Another dynamical property of the VPFN system related to its excitable character is that, for a periodic stimulation of fixed amplitude, the pulse-emission efficiency strongly depends on the repetition rate $f$ of the stimuli [23]. By varying $f$ in the range $10-50 \mathrm{kHz}$ (see Fig. 6) it is observed that the efficiency decreases from almost $1(f<18 \mathrm{kHz})$ exhibiting a series of plateaus at $0.5(25 \mathrm{kHz} \lesssim f \lesssim 30 \mathrm{kHz}), \quad 0.35$ ( $35 \mathrm{kHz} \lesssim f \lesssim 42 \mathrm{kHz}$ ), and so on. This behavior, first found in the VPFN model [23], is due to the phase locking of the excitable system to the train of stimuli. At low frequencies, the response and the stimulation lock 1:1. However, when the period of the stimuli approaches the refractory time, the system can no longer maintain the 1:1 locking, and $50 \%$ of the pulses are then lost, thus leading to 1:2 locking. As the stimulation period is further reduced, the second pulse approaches the refractory time, so only one out of three stimuli induces a response, decreasing the efficiency to $1 / 3$, and so on. This behavior is again in agreement with that of the VPFN model.

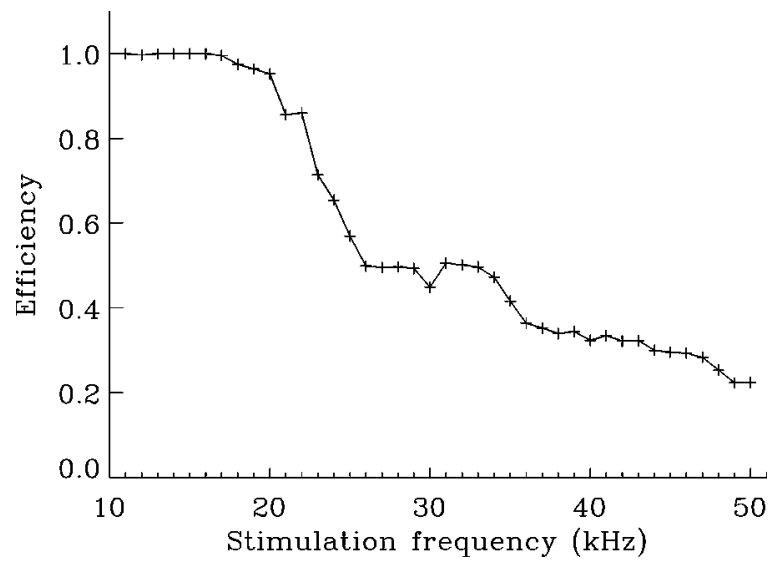

FIG. 6. Pulse emission efficiency when the amplifier bias current is periodically kicked as a function of the kicking frequency.

A phenomenon directly connected to excitability is the so-called coherence resonance [24], for which a maximum ordering of the pulse train is obtained upon addition of a finite level of noise. This counterintuitive phenomenon can be qualitatively understood considering the different dependence of the pulsing rate and the refractory time on noise level. While coherence resonance has been already demonstrated in excitable optical systems $[7,10]$ involving different phase-space features, we expect to observe this behavior also in our system due to its excitable properties. In order to check whether our experimental system displays coherence resonance, we prepare the system in the stable high state and we add broadband electronic noise of variable amplitude (produced by an HP33120A function generator) to the signal driving the acousto-optic modulator. The maximum noise amplitude of $600 \mathrm{mV}$ corresponds to a $1.5 \% \mathrm{rms}$ fluctuation in optical power, i.e., $0.1 \mathrm{~mW}$ compared to the continuous wave injection level of $6.6 \mathrm{~mW}$. The results in the left panels in Fig. 7 show that for both low and high noise levels, the train of excitable pulses is more irregular than for intermediate noise levels. The coherence of the output is characterized by the indicator $R=\sigma_{T} /\langle T\rangle$, where $T$ is the time be-

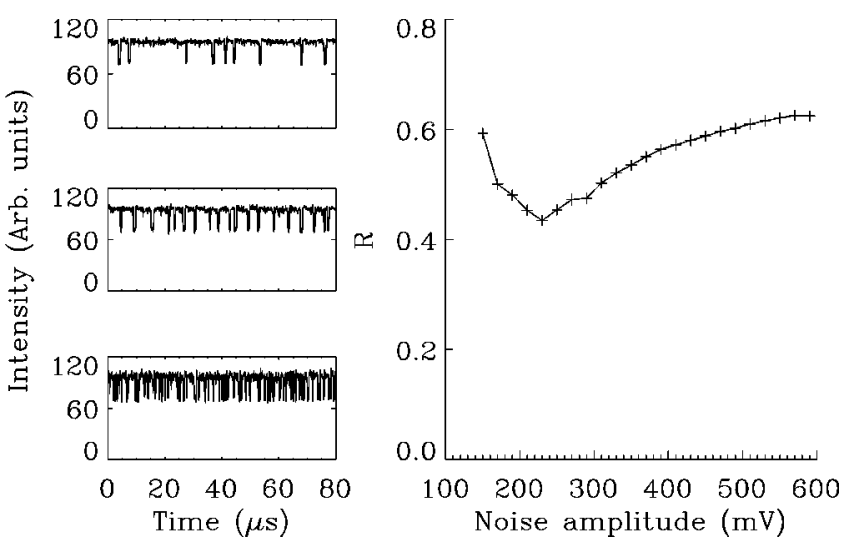

FIG. 7. Left: Time traces of the output power for different levels of added noise. From top to bottom, noise levels are 150, 230, and $400 \mathrm{mV}$, respectively. Right: Dependence of $R$ on the amplitude of the input noise, clearly showing a minimum around $230 \mathrm{mV}$. 

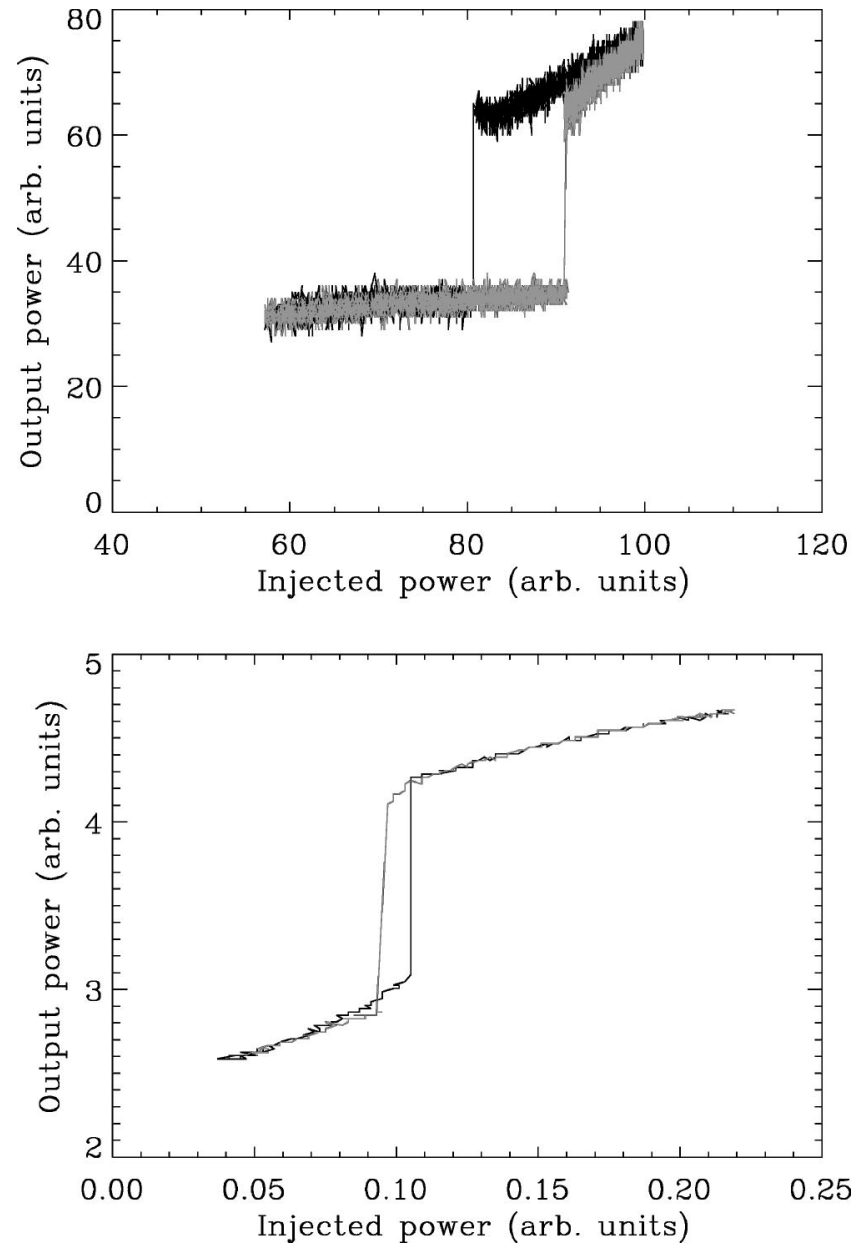

FIG. 8. Output power vs injection power for sweeping frequencies of the injected power of $30 \mathrm{kHz}$ (upper panel) and $60 \mathrm{kHz}$ (lower panel). The black part of the trace corresponds to increasing the injected power, while the gray part corresponds to decreasing it.

tween pulses, $\langle T\rangle$ is its average value, and $\sigma_{T}=\langle(T$ $\left.-\langle T\rangle)^{2}\right\rangle^{1 / 2}$ is its standard deviation [24]. As shown in Fig. 7, $R$ displays a clear minimum when the amplitude of the added noise is around $230 \mathrm{mV}$, clearly indicating the existence of coherence resonance as expected in the VPFN scenario.

Finally, in Fig. 8 we show the output power vs the injected power when the latter is modulated at two different sweeping frequencies $f$. At first glance these curves look like a hysteresis cycle typical of bistable systems. Closer inspection reveals that the opening of one cycle depends on $f$, and that when $f<f_{C}$, the direction of the cycle is reversed [see Fig. 8(b)], and therefore it cannot be interpreted in the framework of bistability. Instead, these results are in perfect agreement with the behavior of the VPFN system under the modulation of a parameter across the unstable region (see Fig. 9). It can be seen that the apparent hysteresis cycle is reversed upon increasing the modulation frequency, and the cycle area depends strongly on the modulation frequency. The reason is that the self-sustained oscillations phase lock to the sweeping
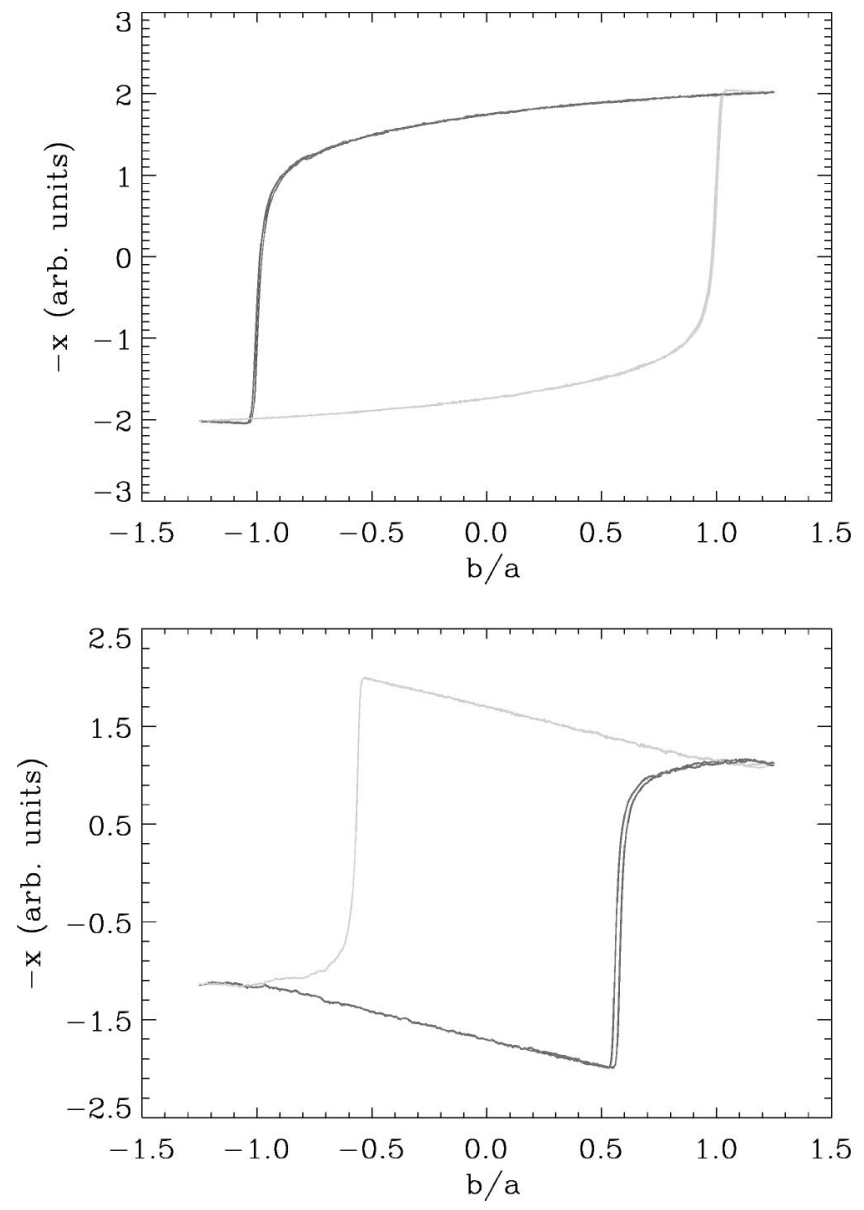

FIG. 9. The fast variable $x$ in the VPFN model as a function of parameter $b$ when it is modulated from $b / a=-1.25$ to $b / a=1.25$ with a period of 220 time units (upper panel) and 318 time units (lower panel). The black part of the trace corresponds to increasing $b$, while the gray part corresponds to decreasing it. Other parameters as in Fig. 3.

signal with a phase lag that may be either positive or negative depending on the ratio $f / f_{C}$ : reversed cycles are obtained when $f<f_{C}$ and otherwise, and moreover, the cycle opening depends on $f$. This provides an easy explanation for previously reported reversed hysteresis cycles $[20,25]$.

As a conclusion, we have experimentally shown that thermo-optical pulsation in broad area semiconductor optical amplifiers can be dynamically interpreted within the scenario of the van der Pol-Fitzugh-Nagumo model. We have demonstrated excitable, oscillatory, phase-locking and coherence resonance behaviors as the intensity of either the injected optical field or the bias current are varied, modulated, or perturbed with additional noise, in good agreement with the theoretical predictions made for this model.

We acknowledge financial support from the CICYT, Grant No. TIC99-0645-C05-02 (Spain) and from the E.U. project PIANOS (ESPRIT 28235). 
[1] A.L. Hodgkin and A.F. Huxley, J. Physiol. (London) 117, 500 (1952)

[2] J.D. Murray, Mathematical Biology (Springer, New York, 1990).

[3] Irving R. Epstein and John A. Pojman, An Introduction to Nonlinear Chemical Dynamics: Oscillations, Waves, Patterns and Chaos (Oxford University Press, New York, 1998).

[4] B. van der Pol, Philos. Mag. 3, 65 (1927).

[5] D.E. Postnov, S.K. Han, T.G. Yim, and O.V. Sosnovtseva, Phys. Rev. E 59, R3791 (1999).

[6] M. Giudici, C. Green, G. Giacomelli, U. Nespolo, and J.R. Tredicce, Phys. Rev. E 55, 6414 (1997).

[7] G. Giacomelli, M. Giudici, S. Balle, and J.R. Tredicce, Phys. Rev. Lett. 84, 3298 (2000).

[8] F. Plaza, M.G. Velarde, F.T. Arecchi, S. Boccaletti, M. Ciofini, and R. Meucci, Europhys. Lett. 38, 85 (1997).

[9] See J.R. Tredicce, in Fundamental Issues of Nonlinear Laser Dynamics: Concepts, Mathematics, Physics and Applications, edited by B. Krauskopf and D. Lenstra, AIP Conf. Proc. No. 548 (AIP, Melville, NY, 2000), and references therein.

[10] H.J. Wünsche, O. Brox, M. Radziunas, and F. Hennenberger, Phys. Rev. Lett. 88, 023901 (2002).

[11] R.A. FitzHugh, Biophys. J. 1, 445 (1961); J.S. Nagumo, S. Arimoto, and S. Yoshizawa, Proc. IREE Austr. 50, 2061 (1962).

[12] Y.A. Rzhanov, H. Richardson, A.A. Hagberg, and J.V. Moloney, Phys. Rev. A 47, 1480 (1993).
[13] W. Lu, D. Yu, and R.G. Harrison, Phys. Rev. A 58, R809 (1998); Opt. Lett. 24, 578 (1999).

[14] H.M. Gibbs, Optical Bistability: Controlling Light with Light (Academic Press, Orlando, 1985); S.D. Smith, Philos. Trans. R. Soc. London, Ser. A 313, 187 (1984).

[15] R. Bonifacio and L. Lugiato, Lett. Nuovo Cimento Soc. Ital. Fis. 21, 510 (1978).

[16] J.L. Jewell, H.M. Gibbs, S.S. Tang, A.C. Gossard, and W. Wiegmann, Appl. Phys. Lett. 40, 291 (1982).

[17] E. Abraham, Opt. Commun. 61, 282 (1987).

[18] H.A. MacKenzie, J.J.E. Reid, H.A. Al-Attar, and E. Abraham, Opt. Commun. 60, 181 (1986).

[19] P. Suret, D. Derozier, M. Lefranc, J. Zemmouri, and S. Bielawski, Phys. Rev. A 61, 021805(R) (2000).

[20] S. Sivaprakasam, D. Narayana Rao, and Ranjit S. Pandher, Opt. Commun. 176, 191 (2000).

[21] M. Brambilla, L.A. Lugiato, F. Prati, L. Spinelli, and W.J. Firth, Phys. Rev. Lett. 79, 2042 (1997).

[22] A.A. Andronov, A.A. Vitt, and S.E. Khaikin, Theory of Oscillators (Dover, New York, 1987), pp. 342-350.

[23] M. Feingold, D.L. González, O. Piro, and H. Viturro, Phys. Rev. A 37, 4060 (1988).

[24] A. Pikovsky and J. Kurtz, Phys. Rev. Lett. 78, 775 (1997).

[25] T. Ackemann, S. Barland, M. Giudici, J.R. Tredicce, S. Balle, R. Jaeger, M. Grabherr, M. Miller, and K.J. Ebeling, Phys. Status Solidi B 221, 133 (2000). 